\title{
Risk factors for ischaemic heart disease: the prospective phase of the British Regional Heart Study
}

\author{
A G SHAPER,${ }^{1} \mathrm{~S}$ J POCOCK,${ }^{1} \mathrm{M}$ WALKER, ${ }^{1}$ A N PHILLIPS, ${ }^{1} \mathrm{~T}$ P WHITEHEAD, ${ }^{2}$ AND \\ P W MACFARLANE ${ }^{3}$ \\ From the Department of Clinical Epidemiology and General Practice, ${ }^{1}$ Royal Free Hospital School of Medicine, \\ London NW3 2PF; Department of Clinical Chemistry, ${ }^{2}$ Wolfson Research Laboratories, Queen Elizabeth \\ Medical Centre, Birmingham; and University Department of Medical Cardiology, ${ }^{3}$ Royal Infirmary, Glasgow
}

SUMMARY Risk factors for major ischaemic heart disease (acute myocardial infarction or sudden death) have been investigated in a prospective study of 7735 men aged 40-59 years drawn from general practices in 24 British towns. After a mean follow-up of $4 \cdot 2$ years, there have been 202 cases of major ischaemic heart disease. Univariate estimates of the risk of ischaemic heart disease show that serum total cholesterol, HDL-cholesterol and triglyceride concentrations, systolic and diastolic blood pressures, cigarette smoking, and body mass index are all associated with increased risk of ischaemic heart disease. Evidence of ischaemic heart disease at initial examination is also strongly associated with increased risk of subsequent ischaemic heart disease.

All these factors were then considered simultaneously using multiple logistic models. Definite myocardial infarction on electrocardiogram and recall of a doctor diagnosis of ischaemic heart disease remained predictive of subsequent major ischaemic heart disease, after allowance for all other risk factors. Serum total cholesterol, blood pressure, and cigarette smoking each remained as highly significant independent risk factors whereas overweight, above average levels of HDL-cholesterol and serum triglyceride were not predictive of risk after allowance for the above factors.

Men with and without pre-existing ischaemic heart disease were examined separately in the same way (using multiple logistic models). The strength of association between the principal risk factors and subsequent major ischaemic heart disease was reduced in the men with pre-existing ischaemic heart disease, only age and serum total cholesterol remaining highly significant.

Overall the levels of the major risk factors commonly encountered in British men have a marked effect on the risk of ischaemic heart disease. Modification of these risk factors in the general population constitutes an important national priority.

For many middle-aged men the onset of ischaemic heart disease, as manifested by angina, acute myocardial infarction or sudden death, is not a completely unexpected phenomenon, arriving 'out of the blue' with no warning to the community or the individual. There is a wealth of evidence to indicate that there are personal characteristics which are strongly associated with increased risk of ischaemic heart disease in human populations, and these 'risk factors' can be determined for both individuals and communities. ${ }^{12}$ However, there is little information in Great Britain on the distribution of the risk factors for ischaemic heart disease in the population. Also, it is not certain to what extent the marked regional variations in ischaemic heart disease mortality can be attributed to variations in established risk factors. ${ }^{3}$ There is little agreement as to the relative importance of the various risk factors or the level of each factor at which risk of ischaemic heart disease becomes increased.

The prospective phase of the British Regional Heart Study aims to determine the role of risk factors, individual and environmental, in middle-aged British men drawn from the general population through general practice registers and to assess the relative importance of the different risk factors. The study is also concerned to determine whether such factors continue to exert an effect on 
risk in those who have already manifest ischaemic heart disease. This presentation is concerned with those risk factors regarded as 'well established'; later publications will deal with other potential risk factors which are less well established, for example, alcohol intake, social class, and personality.

\section{Subjects and methods}

The British Regional Heart Study examined 7735 men aged 40-59 years randomly selected from the age-sex registers of group general practices in 24 towns in England, Wales, and Scotland. The criteria for selecting the towns, the general practices, and the subjects, as well as the methods of data collection, have been presented in previous reports. ${ }^{45}$ In brief, the 24 towns were primarily selected from those with populations of 50-100 000 (1971 census). They were chosen to represent the full range of cardiovascular disease mortality and included towns in all the major standard regions. The general practice selected in each town was required to have a social class distribution representative of the men of that town. The men were selected at random from age-sex registers; no attempt was made to exclude subjects with cardiovascular problems and there was a $78 \%$ response rate. Research nurses administered a questionnaire and completed an examination of each man, including an electrocardiogram. ${ }^{5}$

\section{BLOOD PRESSURE}

The London School of Hygiene sphygmomanometer was used to measure the blood pressure twice in succession with the subject seated and the arm supported on a cushion. Diastolic blood pressure was recorded at disappearance of sounds (phase V). All blood pressure readings were adjusted for observer variation within each town in order to allow for inconsistencies between the three observers.

\section{SMOKING}

Cigarette smoking habits derived from a standardised questionnaire were as follows: never smoked cigarettes, ex-cigarette smoker, and current cigarette smoker at four levels $(1-19,20,21-39$, $\geqslant 40$ per day). Ex-cigarette smokers who currently smoked pipe/cigars are included in 'ex-cigarette smokers'. Those men who had only ever smoked pipe/cigars are included in 'never smoked cigarettes'.

BODY MASS INDEX

This was defined as weight/height ${ }^{2}$.

SERUM LIPIDS

Detailed information on blood lipid measurements has been published. ${ }^{6}$ Adjustments have been made for the marked diurnal variation in triglyceride measurement. Triglyceride concentrations have a highly skewed distribution, so log transformation and geometric means have been used.

PRE-EXISTING ISCHAEMIC HEART DISEASE

The presence of ischaemic heart disease at initial examination was determined by an administered questionnaire enquiring into chest pain and by electrocardiogram. In addition, subjects were asked whether a doctor had ever told them that they had any form of cardiovascular disease. The methodology has been fully described in previous publications. ${ }^{57}$

For the purposes of this paper 'definite angina' and 'possible angina' have been combined as 'angina'. Any subject who could recall a diagnosis by a doctor of angina or heart attack (myocardial infarction, coronary thrombosis) was considered to have recall of ischaemic heart disease.

MISSING VALUES

Triglyceride estimations were not made in the first six towns so the triglyceride comparison is based on 14 cases and 5535 other men. The number of missing values for other factors were as follows: cigarette smoking-16, systolic blood pressure-8, diastoli요응 blood pressure -10 , body mass index -3 , serum tota cholesterol-45, HDL cholesterol-315. There are $s$ missing data for the chest pain questionnaire in $16 \frac{\mathrm{D}}{0}$ men, electrocardiogram in 8 men, and diagnosis-recall in 11 men. One man with a missing chest pain questionnaire became a case of ischaemico. heart disease. Therefore, in table 5 , the number of cases is 201 and not 202, and in table 7 there are only 201 cases.

FOLLOW-UP PROCEDURES

All the 7735 men who took part in the initial examination in 1978-80 are being followed for both morbidity and mortality over at least five years. To date, follow-up has been achieved on $99 \%$ of the original cohort. Full details of the follow-up procedures have been published. ${ }^{8}$

CASE DEFINITION FOR MAJOR ISCHAEMIC HEART DISEASE

The following definitions were used to determine whether any reported cardiovascular event during follow-up could be regarded as 'a case' of major ischaemic heart disease for inclusion in this study.

Fatal cases

Any subject for whom the death certificate recorded ischaemic heart disease (ICD 410-414) as the cause of death and for whom this statement was not contradicted by the medical history or postmortem 
examination. Sudden death for which no other cause was apparent, and which had been certified as due to ischaemic heart disease, was included in this category. In six subjects included as cases the death certificates were not coded ICD 410-414. The manner in which these death certificates were completed led to enquiries which supported a diagnosis of ischaemic heart disease as the cause of death. Where ischaemic heart disease was recorded on the death certificate but was not regarded as the cause of death, as in cases with cancer or pulmonary embolus, the subject was not included as a case. There were five such instances.

\section{Non-fatal cases}

Any report of myocardial infarction which included at least two of the following manifestations:

(a) preceded by severe prolonged chest pain;

(b) electrocardiographic evidence of myocardial infarction;

(c) cardiac enzyme changes associated with myocardial infarction.

\section{Results}

\section{GENERAL FINDINGS}

As of July 1983, $202(2 \cdot 6 \%)$ of the men had experienced an acute myocardial infarction or sudden death attributed to ischaemic heart disease since they were examined by the Regional Heart Study team. The mean period of follow-up thus far is 4.2 years and the overall attack rate of major ischaemic heart disease is 6.2 per 1000 men per year. The age distribution of these 202 cases of major ischaemic heart disease (table 1) shows the anticipated age trend. Men aged 55-59 were more than four times as likely to become a case as men aged $40-44$. Eighty-one (40\%) of the 202 cases were fatal and 45 of these died in less than one hour from onset of symptoms.

The following results compare the 202 cases of major ischaemic heart disease with the other 7533 men as regards (1) established risk factors for ischaemic heart disease, and (2) evidence of ischaemic heart disease at the initial examination.

Table 1 Age distribution of 202 cases of major ischaemic heart disease occurring in an average of $4 \cdot 2$ years

\begin{tabular}{lll}
\hline Age $(y r)$ & No of cases/total no of men $(\%)$ \\
\hline $40-44$ & $16 / 1838 \quad(0 \cdot 9)$ \\
$45-49$ & $47 / 1898 \quad(2 \cdot 5)$ \\
$50-54$ & $57 / 1974 \quad(2 \cdot 9)$ \\
$55-59$ & $82 / 2025 \quad(4 \cdot 0)$ \\
& $202 / 7735$ \\
\hline
\end{tabular}

\section{(1) RISK FACTORS}

\section{Overall findings}

For this first presentation of the prospective results we confine attention to those risk factors that previous studies have shown to be closely associated with increased risk of major ischaemic heart disease: cigarette smoking, systolic and diastolic blood pressure, body mass index (weight/height ${ }^{2}$ ), serum total cholesterol, serum triglyceride, and high density lipoprotein-cholesterol (HDL-cholesterol). A univariate approach is used as a preliminary description of the basic results. However, in order to allow for interrelations between risk factors, multivariate analysis is used in later sections.

Table 2 shows the pattern of cigarette smoking for cases and other men. The percentage of men who never smoked cigarettes was considerably lower in cases $(p=0 \cdot 0001)$. For the other (quantitative) risk factors the mean levels for cases and other men have been compared (table 3). Systolic blood pressure, diastolic blood pressure, body mass index, serum total cholesterol, and serum triglyceride all showed higher mean levels while HDL-cholesterol showed a lower mean level in cases. All variables showed a highly significant difference between cases and other men ( $p=0.001$ for each two-sample $t$ test) in the direction anticipated from previous studies. Of the risk factors, only systolic blood pressure showed any marked association with age $(r=+0 \cdot 21)$. However, the simple comparisons in table 3 are largely unaffected by the fact that cases are on average some two years older than the other men.

Table 2 Cigarette smoking in cases and other men

\begin{tabular}{lrl}
\hline Cases & & Other men \\
\hline $\begin{array}{l}\text { Cigarette smoking } \\
\text { Never }\end{array}$ & $18(9 \%)$ & $1801(24 \%)$ \\
Ex & $76(38 \%)$ & $2639(35 \%)$ \\
Current & $108(53 \%)$ & $3077(41 \%)$ \\
Total & $202(100 \%)$ & $7517(100 \%)$ \\
\hline
\end{tabular}

Table 3 Means of quantitative risk factors in cases and other men

\begin{tabular}{|c|c|c|c|}
\hline & $\begin{array}{l}\text { Cases } \\
(n=202)\end{array}$ & $\begin{array}{l}\text { Other men } \\
(n=7533)\end{array}$ & t-value \\
\hline Age (years) & $52 \cdot 8$ & $50 \cdot 2$ & $7 \cdot 1$ \\
\hline Systolic BP (mm Hg) & $155 \cdot 4$ & $144 \cdot 9$ & $6 \cdot 1$ \\
\hline Diastolic BP (mm Hg) & $87 \cdot 9$ & $82 \cdot 1$ & $5 \cdot 1$ \\
\hline Body mass index $\left(\mathrm{kg} / \mathrm{m}^{2}\right)$ & 26.44 & $25 \cdot 46$ & $4 \cdot 1$ \\
\hline Total cholesterol $(\mathrm{mmol} / \mathrm{l})$ & $6 \cdot 78$ & $6 \cdot 29$ & $6 \cdot 0$ \\
\hline Triglyceride ${ }^{*}(\mathrm{mmol} / \mathrm{l})$ & $2 \cdot 10$ & 1.73 & $4 \cdot 1$ \\
\hline HDL-C $(\mathrm{mmol} / \mathrm{l})$ & $1 \cdot 08$ & $1 \cdot 15$ & $-3 \cdot 5$ \\
\hline
\end{tabular}


Risk and the individual (univariate analysis)

It is necessary to consider how an individual's chance of becoming a case of major ischaemic heart disease is associated with his observed risk factor levels. The unadjusted results shown in the figure are based on the following statistical approach. For each quantitative risk factor, the men were ranked in order of magnitude of the measurement and then divided into five groups of approximately equal size. For instance, body mass index has groups $<22.89$, $22 \cdot 89-24 \cdot 57,24 \cdot 58-26 \cdot 01,26 \cdot 02-27 \cdot 91$, and $\geqslant 27.92 \mathrm{~kg} / \mathrm{m}^{2}$, containing $1550,1545,1540,1551$, and 1546 men respectively. The number of ischaemic heart disease cases in each group (eg, for the above body mass index groups $30,26,38,47$, and 61 cases respectively) has then been used to calculate the odds of becoming a case for men in each group. These odds have then been expressed relative to men in the lowest of the five groups. For example, the relative odds for men with body mass index $27.92 \mathrm{~kg} / \mathrm{m}^{2}$ is $2 \cdot 1$, which means that men in the top fifth of body mass apparently have over twice the risk of major ischaemic heart disease compared with men in the bottom fifth of body mass. Lastly, these relative odds have been adjusted for age differences in the five groups (using a logistic model). These age corrections made little difference to the results. The relative odds for HDL-cholesterol groups was expressed relative to the top fifth since HDL-cholesterol is inversely related to risk.

Cigarette smoking Men who have never smoked have a clearly reduced risk relative to all other categories. There is no dose-relation seen between the number of cigarettes smoked and the risk of major ischaemic heart disease. The lower risk in the small group of very heavy smokers ( $\geqslant 40 /$ day) is not significantly different from that in other cigarette smokers. The twofold risk in ex-smokers may in part be because some men have recently given up smoking for reasons of ill health.

Systolic and diastolic blood pressure For systolic blood pressure there is an estimated doubling of risk for the top $40 \%$ of men (ie, systolic blood pressure $\geqslant 148 \mathrm{mmHg}$ ) but there is no evidence of any trend at lower levels. For diastolic blood pressure there is an estimated threefold increase in risk of major ischaemic heart disease in the top fifth (ie, diastolic blood pressure $\geqslant 93 \mathrm{mmHg}$ ) relative to the bottom fifth $(<72 \mathrm{mmHg})$ while the rest $(72-92 \mathrm{mmHg})$ are at intermediate risk.

Body mass index There is a doubling of risk in the top fifth of body mass index relative to the bottom fifth. Note that the difference in weight here is of the order of $20 \mathrm{~kg}$ or 3 stone. There is some indication of a more modest increase in risk for men with lesser elevations in body mass.

Serum total cholesterol There is a continuous and marked trend of increased risk as total cholesterol increases. Men in the top fifth of cholesterol have over three times the risk of men in the bottom fifth. Also, men within the middle range of total cholesterol (6-6.4 mmol/l) have almost double the risk of men in the bottom fifth, this difference being statistically significant $(p=0 \cdot 02)$.

$H D L$-cholesterol Men in the bottom fifth of $\frac{0}{0} \vec{\theta}$ HDL-cholesterol, ie, $<0.93 \mathrm{mmol} / \mathrm{l}$, have double the $\stackrel{\mathrm{\Phi}}{\mathrm{D}}$ 을 risk of major ischaemic heart disease compared with $\vec{\Phi} \rightarrow$ all other men. However, above $0.93 \mathrm{mmol} / \mathrm{l}$ there is no clear association of HDL-cholesterol with risk.

Serum triglyceride The most striking finding here is that men in the bottom fifth of triglyceride (ie, $<1.08 \mathrm{mmol} / \mathrm{l}$ ) have an estimated risk less than one? third that of all other men. Men in the top fifth $(>2.80 \mathrm{mmol} / \mathrm{l})$ have the highest estimated risk but there is no clear trend with intermediate levels.

\section{Interrelations between risk factors}

Correlations between the six quantitative risk factors for all men in the study are shown in table 4 . Systolic and diastolic blood pressure are the most strongly correlated $(\mathrm{r}=+0 \cdot 70)$. Body mass index has a highly significant positive association with blood pressure, total cholesterol, and triglyceride and a clear negative association with HDL-cholesterol. HDL-cholesterol

Table 4 Correlations between risk factors (for all men)

\begin{tabular}{|c|c|c|c|c|c|c|}
\hline & Systolic BP & Diastolic BP & $B M I$ & Cholesterol & Triglyceride* & $H D L-C$ \\
\hline $\begin{array}{l}\text { Age } \\
\text { Systolic BP } \\
\text { Diastolic BP } \\
\text { Body mass index } \\
\text { Cholesterol } \\
\text { Triglyceride* }\end{array}$ & $0 \cdot 21$ & $\begin{array}{l}0.06 \\
0.70\end{array}$ & $\begin{array}{l}0.03 \\
0.27 \\
0.37\end{array}$ & $\begin{array}{l}0.01 \\
0.08 \\
0.11 \\
0.17\end{array}$ & $\begin{array}{l}0.00 \\
0 \cdot 12 \\
0 \cdot 12 \\
0 \cdot 30 \\
0.37\end{array}$ & $\begin{array}{l}-0.00 \\
-0.01 \\
-0.04 \\
-0.25 \\
-0.04 \\
-0.46\end{array}$ \\
\hline
\end{tabular}

\footnotetext{
* Log transform used
} 
Risk factors for ischaemic heart disease: the prospective phase of the British Regional Heart Study
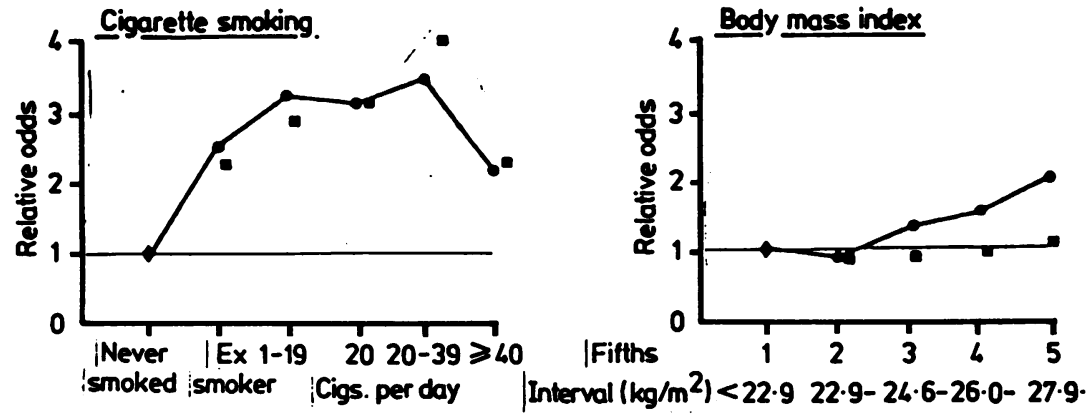

\begin{tabular}{llllllll|llllll} 
No of cases & 18 & 76 & 42 & 28 & 31 & 7 & |No of cases & 30 & 26 & 38 & 47 & 61
\end{tabular}

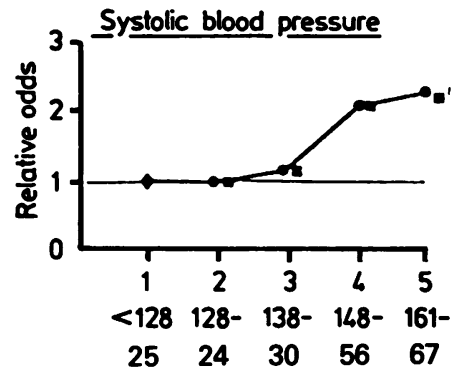

Fifths
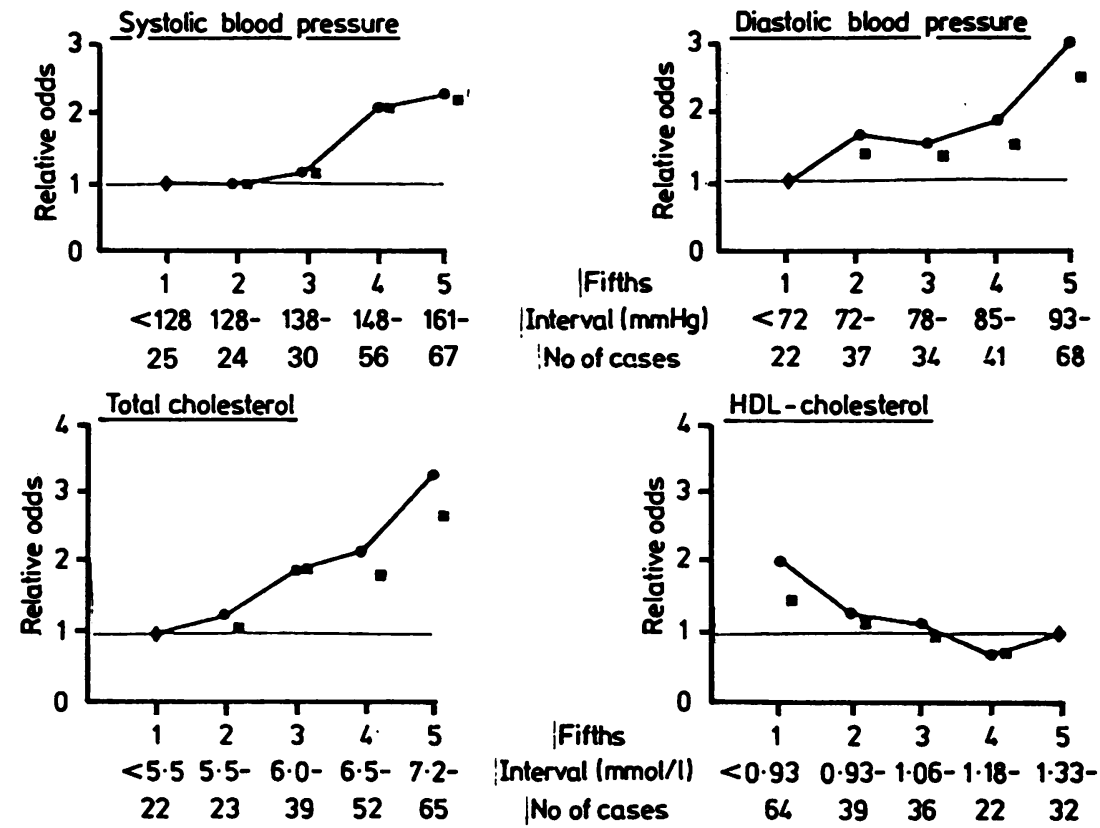

$\begin{array}{cccccc}\text { IInterval (mmHg) } & <72 & 72- & 78- & 85- & 93- \\ \text { No of cases } & 22 & 37 & 36 & 41 & 68\end{array}$

|Fitths
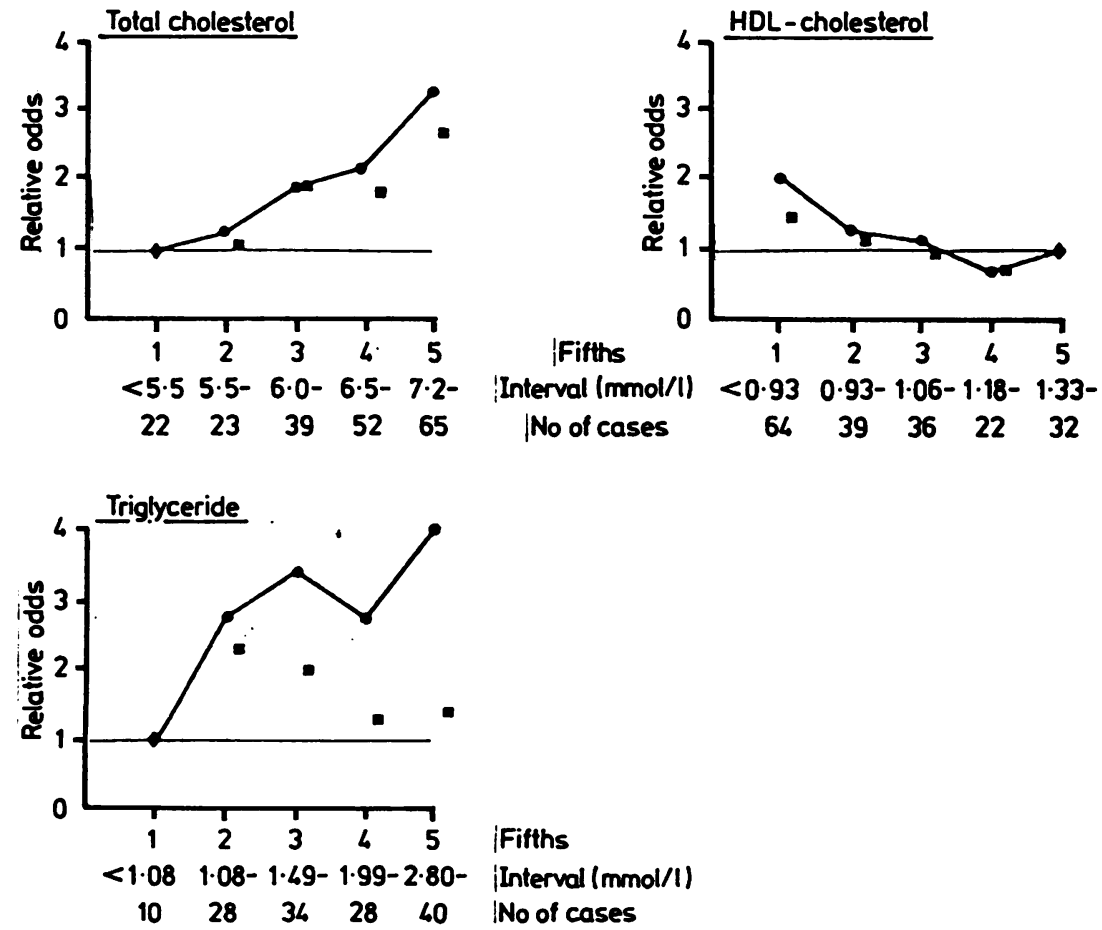

Relative odds for each principal risk factor (by fifths of ranked distribution)

Base group

Unadjusted (except for age)

Adjusted for other risk factors, pre-existing ischaemic heart disease and age. 
and triglyceride have a strong negative association $(r=-0 \cdot 46)$ while total cholesterol and triglyceride are positively correlated $(r=+0 \cdot 37)$. There is no association between HDL-cholesterol and total cholesterol.

The association between cigarette smoking and other risk factors, including age, has been examined (table available on request). On the whole, differences between current smokers and men who have never smoked cigarettes tend to be small, although such differences are statistically significant, because of the large numbers of men. Thus current smokers have slightly lower mean body mass, diastolic blood pressure, and HDL-cholesterol and slightly higher mean age, systolic blood pressure, and serum triglyceride. None of these associations is strong enough to affect the simple increase in risk for current smokers observed in the figure. Compared with non-smokers, ex-smokers tend to be older and have higher mean systolic blood pressure, body mass index, total cholesterol, and triglyceride.

Overall, this complex pattern of interrelations between risk factors requires multivariate analysis in order to estimate the independent contribution of each factor to risk of major ischaemic heart disease after allowance for all other factors. However, it is first necessary to examine the influence of the presence of ischaemic heart disease at the time of initial screening on the risk of subsequent major ischaemic heart disease.

(2) PRE-EXISTING ISCHAEMIC HEART DISEASE There are three sources of information on the presence of ischaemic heart disease at initial screening: a standardised chest pain questionnaire, a computerised three orthogonal lead electrocardiogram, and recall of doctor diagnoses by each man. ${ }^{5}$ From the questionnaire, men could be identified as having angina and/or possible myocardial infarction.

Electrocardiographic abnormalities considered here are myocardial infarction (definite or possible) or myocardial ischaemia (definite or possible). Recall of doctor diagnosis is confined to angina and/or myocardial infarction.

Table 5 shows the distributions of cases and other men for each of the three types of pre-existing ischaemic heart disease. The diagnostic categories are not exclusive, and the patterns of overlap have been fully described in earlier publications. ${ }^{57}$ In all, 1940 men $(25 \%)$ had at least one indicator of pre-existing ischaemic heart disease. All indicators of ischaemic heart disease prevalence (except possible myocardial infarction on electrocardiogram, a very small group of men) are significantly associated with an increased risk of subsequent major ischaemic
Table 5 Risk of subsequent major ischaemic heart disease according to pre-existing ischaemic heart disease at initial screening

\begin{tabular}{llrrl}
\hline $\begin{array}{l}\text { Type of } \\
\text { information }\end{array}$ & $\begin{array}{l}\text { Indication } \\
\text { of pre-existing IHD }\end{array}$ & Cases & $\begin{array}{l}\text { Other } \\
\text { men }\end{array}$ & $\begin{array}{l}\text { Relative odds } \\
\text { of subsequent } \\
\text { major IHD } \\
\text { (age-adjusted) }\end{array}$ \\
\hline Questionnaire & None & 131 & 6494 & 1 \\
& Angina only & 22 & 369 & 2.54 \\
& Possible MI only & 21 & 466 & 2.06 \\
& Angina and possible MI & 27 & 189 & 5.96 \\
ECG & None & 128 & 6473 & 1 \\
& Possible ischaemia & 22 & 519 & 1.98 \\
& Definite ischaemia & 16 & 241 & 3.06 \\
& Possible MI & 2 & 84 & 1.01 \\
Definite MI & 34 & 208 & 6.96 \\
Recall of & None & 148 & 7154 & 1 \\
doctor & Angina only & 13 & 118 & 5.33 \\
diagnosis & MI only & 26 & 145 & 8.67 \\
& Angina and MI & 15 & 105 & 6.91 \\
\hline
\end{tabular}

MI = myocardial infarction

heart disease, as indicated in the column of age adjusted relative odds.

\section{Questionnaire}

Positive replies to chest pain questionnaire criteria for angina or possible myocardial infarction are associated with a doubling of risk of subsequent major ischaemic heart disease compared with men who do not reply positively. If both angina and possible myocardial infarction are positive on questionnaire the estimated risk increases sixfold.

\section{Electrocardiogram}

Definite indications of myocardial infarction on electrocardiogram are associated with a sevenfold increase in risk of subsequent major ischaemic heart disease. Possible and definite ischaemia on electrocardiogram (unaccompanied by electrocardiographic evidence of infarction) are associated with two and threefold increases in risk respectively. From here onward, possible infarction and definite and possible myocardial ischaemia are combined into a single category of electrocardiographic abnormality.

\section{Recall of doctor diagnosis}

Men who can recall a doctor diagnosis of ischaemic heart disease have a substantially increased risk of subsequent major ischaemic heart disease. It also appears relatively unimportant whether such recall concerns angina, myocardial infarction or both, so that from now on recall of angina and/or myocardial infarction will be referred to as recall of ischaemic heart disease. 
MULTIVARIATE ANALYSIS

Combined effect of risk factors and pre-existing ischaemic heart disease

The simultaneous influence of risk factors, presence of ischaemic heart disease at initial screening, and age on risk of subsequent major ischaemic heart disease is now considered in all men. Multiple logistic regression has been used to assess the independent contribution of each factor to the risk of major ischaemic heart disease as shown in table 6 .

Table 6 Results of a multiple logistic regression relating risk of major ischaemic heart disease to pre-existing ischaemic heart disease, age, and risk factors (excluding triglyceride) for 7359 men with complete data

\begin{tabular}{lcr}
\hline & $\begin{array}{l}\text { Logistic } \\
\text { coefficient }\end{array}$ & \multicolumn{1}{c}{ t-value } \\
\hline Recall of IHD (yes/no) & 1.042 & $4.3 \mathrm{p}<0.001$ \\
Definite infarct on ECG (yes/no) & 1.071 & $4.2 \mathrm{p}<0.001$ \\
Ischaemia or possible infarct on ECG (yes/no) & 0.334 & $1.6 \mathrm{p}=0.10$ \\
Angina on questionnaire & 0.441 & $1.8 \mathrm{p}=0.07$ \\
& & \\
Risk factors & & \\
Total cholesterol (mmol/1) & 0.309 & $4.6 \mathrm{p}<0.001$ \\
Mean blood pressure* (mm Hg) & 0.025 & $5.0 \mathrm{p}<0.001$ \\
Current cigarette smoker (yes/no) & 1.168 & $4.2 \mathrm{p}<0.001$ \\
Ex cigarette smoker (yes/no) & 0.824 & $2.9 \mathrm{p}<0.01$ \\
Age (years) & 0.046 & $3.2 \mathrm{p}<0.01$ \\
HDL-cholesterol (mmol/1) & -0.576 & $-1.8 \mathrm{p}=0.07$ \\
Body mass index (kg/m $\left.{ }^{2}\right)$ & 0.016 & $0.6 \mathrm{NS}$ \\
\hline
\end{tabular}

*Mean blood pressure $=($ systolic blood pressure $+2 \times$ diastolic blood pressure)/3

A definite myocardial infarction on electrocardiogram and recall of a doctor diagnosis of ischaemic heart disease remain highly significant predictors of risk after allowance for all other factors. Other electrocardiographic abnormalities (ischaemia or possible myocardial infarction) and angina on questionnaire make smaller contributions to risk which are not statistically significant.

Serum total cholesterol, mean blood pressure (= systolic blood pressure $+2 \times$ diastolic blood pressure/3), and cigarette smoking each remain highly significant independent risk factors. The inverse association of HDL-cholesterol is considerably reduced after allowance for other factors and is no longer statistically significant at the $5 \%$ level. Furthermore, if total cholesterol is replaced by non-HDL-cholesterol in the logistic model, then the coefficient for HDL-cholesterol becomes even less significant $(p=0 \cdot 41)$. There is no evidence that body mass affects risk of major ischaemic heart disease once other factors associated with body mass (eg, serum total cholesterol, blood pressure, and HDL-cholesterol) are taken into account. The possible contribution of serum triglyceride was assessed by a further logistic analysis on the 5546 men with complete data (including triglyceride). The logistic coefficient for triglyceride was close to zero, indicating that the risk association of triglyceride seen in univariate analysis (table 3 ) completely disappears once other factors are taken into account.

Since systolic and diastolic blood pressure have similar strengths of association with risk in these data, it seemed reasonable to use mean blood pressure in the logistic model in table 6. However, similar (but slightly less significant) results are obtained if systolic or diastolic is used instead of mean blood pressure.

Further explanation of table 6 is given in the Statistical Appendix.

\section{Independence of risk factors}

A second approach to the analysis of multiple risk factors is to consider each quantitative factor in five equal-sized groups (as previously done in the univariate analyses in the figure). Multiple logistic analyses can then be used to calculate the relative odds of becoming a case for each fifth of each risk factor after adjustment for other risk factors, pre-existing ischaemic heart disease, and age. These adjusted findings are compared with the unadjusted findings in the figure. Note that triglyceride has not been adjusted for in these analyses since it was not estimated for men in six towns and appears to be relatively unimportant.

Cigarette smoking The associations of current cigarette smoking and ex-cigarette smoking with risk remain virtually unchanged after allowance is made for pre-existing ischaemic heart disease, age, and other risk factors.

Body mass index The results for body mass index provide no evidence that overweight is an independent risk factor after other factors $(\mathrm{eg}$, blood pressure and blood lipids) are taken into account.

Serum total cholesterol The association of serum total cholesterol with risk remains highly significant although it has been slightly reduced after allowance for other factors. It is interesting to note that men in the middle interval for total cholesterol $(6 \cdot 0-6.4 \mathrm{mmol} / \mathrm{l})$ still have an estimated twofold risk compared with men at lower levels of cholesterol.

Blood pressure Systolic and diastolic blood pressure are strongly associated, and hence it seems appropriate not to adjust for diastolic blood pressure when studying the association of systolic blcod pressure with risk of major ischaemic heart disease. Accordingly, the trend for systolic blood pressure with risk in the figure is adjusted for all other factors except diastolic blood pressure. Such adjusted 
relative odds for systolic blood pressure are very similar to the unadjusted pattern. Similarly, the pattern for diastolic blood pressure is largely unaffected by adjustment for other factors.

HDL-cholesterol HDL-cholesterol no longer appears as an important risk factor once other factors are allowed for. There remains some suggestion that the lowest fifth of HDL-cholesterol carries a slightly increased risk but there is no indication that higher values of HDL-cholesterol are protective against ischaemic heart disease. If the analyses are repeated with non-HDL-cholesterol replacing total cholesterol then the adjusted relative odds for HDL-cholesterol $<0.93 \mathrm{mmol} / \mathrm{l}$ are further reduced from 1.47 (as in the figure) to $1 \cdot 26$.

Triglyceride Triglyceride shows an erratic pattern of association with risk after allowance for all other factors. There is no evidence of an increased risk at high levels of triglyceride. The curious elevation in risk for triglyceride in the range $1.08-1.98 \mathrm{mmol} / \mathrm{l}$ is difficult to understand and perhaps is one of those chance findings to be expected in a multivariate analysis of so many factors.
PRE-EXISTING ISCHAEMIC HEART DISEASE AND RISK FACTORS

Three aspects of pre-existing ischaemic heart disease at initial screening (questionnaire, electrocardiogram, and recall of doctor diagnosis) have already been described in table 5. A total of 1940 men $(25 \%)$ had at least one of these indicators of pre-existing disease, and these men have contributed $105(52 \%)$ of the subsequent cases of major ischaemic heart disease.

An important issue is whether the 'established' risk factors continue to exert an influence on the risk of subsequent major ischaemic heart disease in men who already have evidence of existing ischaemic heart disease. When the mean levels of risk factors in cases of major ischaemic heart disease with and without pre-existing ischaemic heart disease are compared, it can be seen that there is remarkably little difference between the two groups (table 7). It can also be seen that cases have higher mean levels of the risk factors (lower for HDL-cholesterol) than other men, both in those with and those without pre-existing ischaemic heart disease.

In table 8 , the simultaneous influence of risk factors on the emergence of major ischaemic heart

Table 7 Means of risk factors for cases and other men by pre-existing ischaemic heart disease at initial screening

\begin{tabular}{|c|c|c|c|c|}
\hline & \multicolumn{2}{|c|}{ Cases of major IHD } & \multicolumn{2}{|l|}{ Other men } \\
\hline & $\begin{array}{l}\text { Pre-existing } \\
\text { IHD } \\
\text { (105 men) }\end{array}$ & $\begin{array}{l}\text { No pre-existing } \\
\text { IHD } \\
\text { ( } 96 \text { men) }\end{array}$ & $\begin{array}{l}\text { Pre-existing } \\
\text { IHD } \\
\text { (1835 men) }\end{array}$ & $\begin{array}{l}\text { No pre-existing } \\
\text { IHD } \\
\text { (5663 men) }\end{array}$ \\
\hline Systolic BP (mm Hg) & 153.9 & $157 \cdot 0$ & $143 \cdot 8$ & $143 \cdot 8$ \\
\hline Diastolic BP (mm Hg) & $88 \cdot 3$ & $87 \cdot 5$ & $83 \cdot 7$ & $81 \cdot 6$ \\
\hline Body mass index $\left(\mathrm{kg} / \mathrm{m}^{2}\right)$ & $26 \cdot 5$ & $26 \cdot 4$ & 25.9 & $25 \cdot 3$ \\
\hline Total cholesterol $(\mathrm{mmol} / \mathrm{l})$ & 6.77 & 6.78 & $6 \cdot 36$ & $6 \cdot 26$ \\
\hline HDL-cholesterol (mmol/l) & 1.06 & $1 \cdot 10$ & $1 \cdot 13$ & $1 \cdot 15$ \\
\hline Triglyceride* $(\mathrm{mmol} / \mathrm{l})$ & 2.09 & $2 \cdot 11$ & 1.82 & $1 \cdot 70$ \\
\hline \% current cigarette smokers & $54 \%$ & $53 \%$ & $44 \%$ & $40 \%$ \\
\hline
\end{tabular}

*Geometric means

Table 8 Results of a multiple logistic regression relating risk of major ischaemic heart disease to age and risk factors for men with and without pre-existing ischaemic heart disease

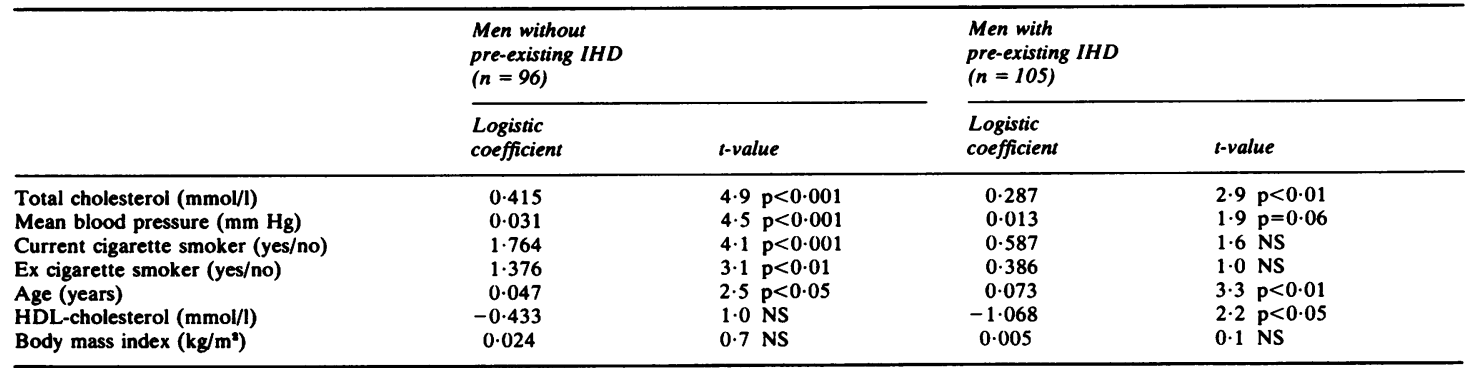


disease events has been determined separately for men with and without pre-existing ischaemic heart disease (using multiple logistic regression as in table 6; see Statistical Appendix). The principal risk factors (age, mean blood pressure, serum total cholesterol, cigarette smoking) are all strongly and independently associated with risk in men without pre-existing ischaemic heart disease. A direct comparison of the logistic coefficients in the two groups provides a clear measure of the extent by which the influence of each risk factor is reduced in those with pre-existing ischaemic heart disease. Only age and serum total cholesterol still have significant coefficients. It seems unusual that HDL-cholesterol appears more strongly associated with risk in men with pre-existing ischaemic heart disease, and this finding will be more fully explored after further follow-up.

\section{Absolute risk level}

The overall annual attack rate of $6 \cdot 2$ per 1000 men can be seen to be made up of an attack rate (incidence) of 4.0 per 1000 in men without pre-existing ischaemic heart disease and 13.6 per 1000 in men with pre-existing ischaemic heart disease.

\section{Discussion}

The British Regional Heart Study provides a unique opportunity to study how risk factors for ischaemic heart disease in a representative sample of British men relate to subsequent risk of major ischaemic heart disease. Previous British prospective studies of ischaemic heart disease have concentrated on occupational groups (The Whitehall study and the UK Heart Disease Prevention Project ${ }^{10}$ ) and have not included men from areas with high mortality, eg, the North of England and Scotland. Recruitment and follow-up of men from general practices spread throughout the country enables us to provide community-based information on risk factors which should be of considerable relevance in understanding the aetiology of ischaemic heart disease and in implementing measures to prevent major ischaemic heart disease events.

In some prospective studies, men with evidence of pre-existing ischaemic heart disease have been excluded from follow-up and analysis, eg, the US Pooling Project. ${ }^{11}$ In view of the high prevalence of ischaemic heart disease in Great Britain, as in many other developed countries, it appears to be important to include all men, irrespective of whether or not they have evidence of pre-existing ischaemic heart disease. This allows for a study of risk factors in relation to the presentation of major ischaemic heart disease in a realistic setting. To exclude the $25 \%$ of middle-aged men with some evidence of pre-existing ischaemic heart disease seems artificial, particularly when it is well established that pre-existing ischaemic heart disease is an extremely important predictor of further major ischaemic heart disease.

\section{RISK FACTORS}

Our principal aim has been to define the impact that elevated levels of commonly accepted risk factors make on the risk of major ischaemic heart disease in British men. We have presented results for each risk factor separately in univariate analysis and have also used multivariate analysis to study their simultaneous and independent contributions to risk of major ischaemic heart disease. The findings for each risk factor are discussed in turn.

Cigarette smoking is a powerful risk factor in that current smokers in this study' have three times the risk of major ischaemic heart disease compared with men who have never smoked cigarettes. This finding is consistent with many other prospective studies. We have not demonstrated any marked dose-response relationship as reported by some others, eg, Pooling Project. ${ }^{11}$ This means that light smokers (less than one pack a day) still carry a considerable excess risk even after allowance for other risk factors. Since nearly half the men in this study were current smokers, the impact of smoking on risk of ischaemic heart disease continues to deserve considerable emphasis. The increased risk in ex-smokers is difficult to interpret, since any decision to stop smoking may have been motivated by preceding ill health. Indeed, recall of doctor diagnosis of ischaemic heart disease at initial screening was several times higher in recent ex-smokers $(<5$ years ago) compared with men who had never smoked cigarettes or those who had given up more than 10 years ago.

Blood pressure is also confirmed as an important independent risk factor for ischaemic heart disease. It has been a long held belief that the diastolic blood pressure is a better indicator of the degree of blood pressure elevation and subsequent risk of cardiovascular disease than systolic blood pressure. The Framingham Study showed clearly that, regardless of the diastolic blood pressure, systolic blood pressure was an excellent predictor of subsequent ischaemic heart disease and that there was good correlation between the two sets of measurements. ${ }^{12}$ In the present study, systolic and diastolic blood pressure appear to be of similar predictive value so that mean blood pressure may be the most appropriate summary statistic. This study 
has demonstrated that the effect of blood pressure on risk of ischaemic heart disease is not solely confined to the commonly accepted criteria of hypertension. For instance, while patients with blood pressure over $160 \mathrm{mmHg}$ carry a considerably increased risk, our data also indicate that subjects with lesser elevations in blood pressure (eg, systolic in the range 148-160 mmHg) still have increased risk compared with subjects with lower blood pressures.

Body mass index is related to risk of ischaemic heart disease but is also associated with several other risk factors, eg, blood pressure and blood lipids. In this study, allowance for these other factors in multivariate analysis indicates that body mass index is not an independent risk factor, a finding supported by other investigators. ${ }^{1314}$ However, there are some prospective studies which claim that obesity (or overweight) is an independent risk factor for ischaemic heart disease, particularly when populations are followed for long periods of time (26 years), ${ }^{1516}$ in younger individuals ${ }^{1617}$ and for sudden death. ${ }^{15} 16$ Even if we can explain most of the mechanisms by which overweight contributes to the risk of ischaemic heart disease, this does not make overweight any more acceptable or benign to those affected by it. For the British population, as in many other developed countries, being overweight carries an increased likelihood of hypertension and hyperlipidaemia as well as an increased risk of diabetes mellitus and many other problems. It is perhaps the most readily detectable sign of increased risk of ischaemic heart disease in our society, and its lack of independent effect is clinically irrelevant.

Serum total cholesterol is confirmed as an independent risk factor whose predictive importance is of a similar order to that of smoking and blood pressure. While hypercholesterolaemia is known to be highly predictive of ischaemic heart disease risk, we wish to stress that men with average British values for serum total cholesterol (ie, 6-6.4 mmol/l) have a significantly increased risk of ischaemic heart disease compared with men having lower cholesterol levels. In population terms, this moderate but continuous elevation in risk through the 'normal range' of serum total cholesterol contributes to many more cases of ischaemic heart disease than does the extreme hypercholesterolaemia that afflicts a small subset of the population. ${ }^{18}$ It should also be noted that lower levels of total cholesterol $(6.0 \mathrm{mmol} / \mathrm{l})$ do not constitute 'no risk' levels, and $45(22 \%)$ of the 202 cases were among men in the lower two fifths of the total cholesterol distribution.

High density lipoprotein-cholesterol (HDL-cholesterol) appears in univariate analysis to have a moderate inverse association with risk (table 3 ) but this is reduced to non-significance after allowance for other factors (see table 6 and figure).

In recent years there has been considerable enthusiasm over the role of HDL-cholesterol as an independent risk factor, albeit in a protective capacity, for ischaemic heart disease. In part, the enthusiasm may have been engendered by an emotional weariness about 'the cholesterol story' and its dietary implications. This study does not support the view that high levels of HDL-cholesterol are protective against ischaemic heart disease. Findings on HDL-cholesterol from other prospective studies have been inconsistent. The most striking effect of HDL-cholesterol was shown in the Troms $\varnothing$ study based on only 17 cases of ischaemic heart disease. ${ }^{19}$ The Framingham study has shown a more moderate effect of HDL-cholesterol which was statistically significant. ${ }^{20}$ However, an Israeli study, ${ }^{21}$ which is the largest previously reported, has shown a rather weak negative association between HDL-cholesterol and risk of ischaemic heart disease which appears to be in agreement with our findings. It is important to recognise that in these middle-aged British men serum total cholesterol is clearly a more important predictor of ischaemic heart disease risk than HDL-cholesterol.

Serum triglyceride does not appear to have any consistent association with risk of ischaemic heart disease once other factors are taken into account, as has previously been indicated by others. ${ }^{22}$

PRE-EXISTING ISCHAEMIC HEART DISEASE In this study, risk of subsequent major ischaemic heart disease was strongly related to two indicators of pre-existing ischaemic heart disease at initial screening, definite myocardial infarction on electrocardiogram and recall of a doctor diagnosis of ischaemic heart disease, even after allowance for risk factors and age. To a lesser extent, myocardial ischaemia on electrocardiogram and evidence of angina using questionnaire also increased the risk of subsequent ischaemic heart disease. Several previous prospective studies have shown the predictive value of electrocardiographic abnormalities even when there is no history of chest pain or overt coronary events. ${ }^{23}{ }^{24} \mathrm{~A}$ man's recall of a doctor diagnosis of ischaemic heart disease has now been shown to be an equally powerful predictor. Admittedly, such information is subjective, depending on a man seeking medical help, being informed of his condition, and being able to recall the fact. However, recall of doctor diagnosis may well be a powerful indicator of the severity of pre-existing ischaemic 
heart disease which cannot be readily obtained by other means.

RISK FACTORS AND PRE-EXISTING ISCHAEMIC HEART DISEASE

An important area of controversy is whether the risk factors continue to exert an influence in those men who have evidence of pre-existing ischaemic heart disease. In the US Coronary Drug Project, serum total cholesterol and cigarette smoking were apparently of little importance in determining long term prognosis after recovery from myocardial infarction, ${ }^{25}$ and it has been said that "the primary risk factors (smoking, serum cholesterol, blood pressure) are of greatest importance in individuals free of signs of coronary heart disease". ${ }^{26}$ The data in table 7, which show similar levels of risk factors in cases of major ischaemic heart disease with and without pre-existing ischaemic heart disease, suggest that risk factors continue to exert an influence in all subjects. However, as pre-existing ischaemic heart disease is a powerful independent predictor of subsequent ischaemic heart disease (see table 6), and as risk factor levels are almost identical in cases with and without pre-existing ischaemic heart disease, then the independent predictive role of the risk factors (total cholesterol, smoking, blood pressure) must be proportionally diminished in those with pre-existing ischaemic heart disease.

Analysis of the data separately for men with and without pre-existing ischaemic heart disease indicates that this is indeed the case (table 8), although with only about 100 cases of major ischaemic heart disease in each group, these findings should be looked upon as a preliminary exploration of the problem. However, as the absolute risk is more than three times higher in men with pre-existing ischaemic heart disease, the established risk factors (total cholesterol, blood pressure) may continue to make an important contribution, albeit proportionally diminished, to the risk of subsequent ischaemic heart disease. This concept is supported by the marked similarity in the levels of risk factors at which major ischaemic heart disease occurs in men both with and without pre-existing heart disease.

ISCHAEMIC HEART DISEASE WITHOUT RISK FACTORS?

Serum total cholesterol, blood pressure, and cigarette smoking are the risk factors of greatest value in predicting the risk of major ischaemic heart disease. There is at least a twofold independent increase in risk associated with any one of the following criteria: (i) serum total cholesterol $\geqslant 6 \mathrm{mmol} / \mathrm{l}$, (ii) systolic blood pressure $\geqslant 148 \mathrm{mmHg}$ or diastolic blood pressure $\geqslant 93 \mathrm{mmHg}$, or (iii) currently smoking cigarettes (figure). Only five of the 202 cases of ischaemic heart disease had none of these risk criteria present and over two-thirds of cases had at least two criteria present. Of the five men with no criteria present, three had evidence of pre-existing ischaemic heart disease. These findings are in contradiction to the statement that "a great many patients, possibly the majority, do not have identifiable risk factors-at least, in the older age groups". ${ }^{27}$ This latter view is perpetuated by a failure to recognise that even moderate elevations in blood lipids and blood pressure carry an excess risk of ischaemic heart disease, especially if the man also smokes cigarettes. For example, a man with serum total cholesterol $\geqslant 6 \mathrm{mmol} / \mathrm{l}$ and systolic blood pressure $\geqslant 148 \mathrm{mmHg}$ who smokes cigarettes has over 10 times the risk of having a major episode of ischaemic heart disease compared with a life-time non-smoker who has lower values of serum total cholesterol and systolic blood pressure.

ANNUAL ATTACK RATE

The annual attack rate of $6 \cdot 2$ per 1000 may be compared with attack rates in the several community heart attack registers. In East London the attack rate for definite and possible myocardial infarction was 10 per 1000 (men 45-64 years). ${ }^{28}$ In Oxford, it was 4.5 per 1000 (men 30-69 years) for confirmed and presumed cases. ${ }^{29}$ In Edinburgh, in men aged 40-59 years the acute heart attack rate was 15 per $1000 .^{30} \mathrm{In}$ all these studies, about half the men had a history of previous ischaemic heart disease. By the nature of the registers, the men had not routinely completed a chest pain questionnaire or had an electrocardiogram before the study. The comparatively low rate of 4 per 1000 observed in the Regional Heart Study men without pre-existing ischaemic heart disease is probably a reasonable estimate of what actually occurs in the middle-aged male British population.

PREDICTION OF A MAJOR ISCHAEMIC HEART DISEASE EVENT

There is considerable interest at present in the ability of the risk factors to predict the development of major ischaemic heart disease in individuals. In the United Kingdom Heart Disease Prevention Project, the probability of major ischaemic heart disease developing in an individual within five years was predicted, using age, blood pressure, plasma cholesterol, smoking habit, physical activity, and body mass index. ${ }^{31}$ Although the risk factors clearly identified a group of subjects at high risk, those within this group who did not manifest major ischaemic heart disease within the five year period were regarded as 'wrongly classified'. This seems an 
unusual approach to a chronic disease in which a wide variety of factors may precipitate a major ischaemic heart disease event in a susceptible subject.

The issue is not whether one can accurately predict the onset of major ischaemic heart disease in a particular individual within a limited period of time, surely an unreasonable expectation in any disease, but whether factors can be identified in a population which considerably increase the risk of major ischaemic heart disease in that population. If these factors can be modified, then there should be a strong incentive to do so. The outcome of a change in risk factor status will depend to a considerable extent on the age at which such a change is made. The presence of lower levels of serum total cholesterol and blood pressure and the absence of cigarette smoking from childhood onward is likely to have a far greater effect on the incidence of ischaemic heart disease than reduction of raised serum total cholesterol and blood pressure and the cessation of cigarette smoking in a middle aged or elderly man.

\section{Statistical appendix}

In table 6 a multiple logistic model was used to assess simultaneously the independent contributions made to the risk of subsequent major ischaemic heart disease by age, the risk factors, and the indicators of pre-existing ischaemic heart disease. This method of multivariate analysis has become standard practice in prospective studies of ischaemic heart disease. ${ }^{32}$ However, to the non-statistical reader some help towards understanding the meaning of logistic coefficients may prove useful. This is most readily explained for the yes/no items such as recall of ischaemic heart disease (yes/no) and current smoker (yes/no). The logistic coefficient, converted to its exponential (ie, antilog), provides an estimate of the relative odds of subsequent major ischaemic heart disease for men in the 'yes' category compared with men in the 'no' category, after the effect of all the other factors on risk have been taken into account. For example, the relative odds for current smokers compared with non-smokers (after allowing for all other risk factors, age and indicators of pre-existing ischaemic heart disease) are given by the exponential of $1 \cdot 168=3 \cdot 2$. Similarly, recall of ischaemic heart disease or a definite myocardial infarction on the ECG each contribute a highly significant threefold increase in the risk of major ischaemic heart disease. However, angina on questionnaire has a relative odds ratio of 1.5 (ie, the exponential of 0.411 ) after allowance for other factors, which is not a significant increase in risk.

For each quantitative risk factor the exponential (antilog) of the logistic coefficient represents the estimated increase in odds of major ischaemic heart disease per unit increase in the measurement. For example, men with serum total cholesterol $=6 \mathrm{mmol} / \mathrm{l}$ have estimated relative odds of 1.36 (exponential of 0.309 ) compared with men having cholesterol $5 \mathrm{mmol} / \mathrm{l}$, after allowance for all other factors. A man with serum total cholesterol of $8 \mathrm{mmol} / \mathrm{l}$ has relative odds of $(1 \cdot 36)^{3}=2 \cdot 5$ compared with a man with serum total cholesterol of $5 \mathrm{mmol} / \mathrm{l}$. The model has assumed that the odds of becoming a case of major ischaemic heart disease increase exponentially with respect to the magnitude of each quantitative risk factor. The model also assumes that all factors contribute independently and multiplicatively to risk of major ischaemic heart disease. The analyses presented in the figure and table 7 go some way towards exploring the validity of these assumptions. However, it remains for further follow-up to provide sufficient cases to explore adequately how the interplay between risk factors and disease prevalence affects the subsequent risk of major ischaemic heart disease.

Lastly, the duration of follow-up for men in the 24 towns ranges from three to five and a half years. Also, the risk of ischaemic heart disease and the reporting $\overrightarrow{0} \overrightarrow{0}$ of cases in the different towns may both depend on $\frac{}{8}$ 'town factors' not incorporated in this analysis. These? two issues could in theory affect the results in this $\vec{D} \overrightarrow{0}$ paper which have ignored between-town differences $\mathbb{D}$ However, the multiple logistic regression has been $\frac{\mathbb{D}}{\mathbb{D}}$ extended to adjust for such town differences (usingo 3 dummy variable for each town), and this produced negligible change in the coefficients for each risk factor. An alternative approach, in which each case was matched with four controls of the same age and from the same general practice, led to results that were comparable to those presented in this paper.

The British Regional Heart Study is supported by a programme grant from the Medical Research Council. Serum total cholesterol and HDL-cholesterol measurements were carried out in the Wolfson Research Laboratories, supported by DHSS. Triglycerides were measured in the Department of Chemical Pathology, Royal Free Hospital School of Medicine under the direction of Dr D G Cramp. Dr Macfarlane's work on computerised electrocardiography is supported by the SHHD and the Greater Glasgow Health Board.

Requests for reprints to: Professor A G Shaper, Department of Clinical Epidemiology and General Practice, Royal Free Hospital School of Medicine, London NW3 2PF 


\section{References}

${ }^{1}$ Dawber TR. The Framingham Study. The epidemiology of atherosclerotic disease. Cambridge, Mass: Harvard University Press, 1980.

${ }^{2}$ Keys A. Seven Countries. A multivariate analysis of death and coronary heart disease. Cambridge, Mass: Harvard University Press, 1980.

${ }^{3}$ Shaper AG. Geographic variations in cardiovascular mortality in Great Britain. Br Med Bull 1984; 40: 366-73.

"Shaper AG, Pocock SJ, Walker M, Cohen NM, Wale CJ, Thomson AG. British Regional Heart Study: cardiovascular risk factors in middle-aged men in 24 towns. Br Med J 1981; 283: 179-86.

${ }^{5}$ Shaper AG, Cook DG, Walker M, Macfarlane PW. Prevalence of ischaemic heart disease in middle-aged British men. Br Heart J 1984; 51: 595-605.

${ }^{6}$ Thelle DS, Shaper AG, Whitehead TP, Bullock DG, Ashby D, Patel I. Blood lipids in middle-aged British men. Br Heart J 1983; 49: 205-13.

${ }^{7}$ Shaper AG, Cook DG, Walker M, Macfarlane PW. Recall of diagnosis by men with ischaemic heart disease. $\mathrm{Br}$ Heart J 1984; 51: 606-11.

${ }^{8}$ Walker M, Shaper AG. Follow-up of subjects in prospective studies based in general practice. $J \boldsymbol{R}$ Coll Gen Pract 1984; 34: 365-70.

${ }^{9}$ Rose G, Reid DD, Hamilton PJS, McCartney P, Keen H, Jarrett RJ. Myocardial ischaemia, risk factors and death from coronary heart disease. Lancet 1977; i: 105-9.

${ }^{10}$ Rose G, Heller RF, Pedoe HT, Christie DGS. The heart disease prevention project: a randomised controlled trial in industry. $\mathrm{Br}$ Med J 1980; 280: 747-51.

${ }^{11}$ The Pooling Project Research Group. Relationship of blood pressure, serum cholesterol, smoking habit, relative weight and ECG abnormalities to incidence of major coronary events. Final report of the Pooling Project. J Chronic Dis 1978; 31: 201-306.

${ }^{12}$ Kannel WB, Gordon T, Schwartz MJ. Systolic versus diastolic blood pressure and risk of coronary disease: The Framingham Study. Amer J Cardiol 1971; 21: 335-46.

${ }^{13}$ Keys AB. Overweight, obesity, coronary heart disease and mortality. Nutrition Today 1980; 15: 16-22.

${ }^{14}$ Larsson B, Svardsudd K, Welin L, Wilhelmsen L, Bjorntorp P, Tibblin G. Abdominal tissue distribution, obesity, and risk of cardiovascular disease and death: 13 year follow-up of participants in the study of men born in 1913. Br Med J 1984; 288: 1401-4.

${ }^{15}$ Hubert HB, Feinleib M, McNamara PM, Castelli WP. Obesity as an independent risk factor for cardiovascular disease: a 26-year follow-up of participants in the Framingham Heart Study. Circulation 1983; 67: 968-77.

${ }^{16}$ Rabkin SW, Mathewson FAL, Hsu PH. Relation of body weight to development of ischaemic heart disease in a cohort of young North American men after a 26 year observation period: the Manitoba Study. Amer J Cardiol 1977; 39: 452-8.
${ }^{17}$ Chapman JM, Coulson AH, Clark VA, Borun ER. The differential effect of serum cholesterol, blood pressure and weight on the incidence of myocardial infarction and angina pectoris. J Chronic Dis 1971; 23: 631-45.

${ }^{18}$ Rose G. Strategy for prevention: lessons from cardiovascular disease. $\mathrm{Br}$ Med J 1981; 282: 1847-51.

${ }^{19}$ Miller NE, Forde OH, Thelle DS, Mjos OD. The Troms $\varnothing$ Heart-Study. High-density lipoprotein and coronary heart-disease: a prospective case-control study. Lancet 1977; i: 965-8.

${ }^{20}$ Gordon TV, Castelli WP, Hjortland MC, Kannel WB, Dawber TR. High density lipoprotein as a protective factor against coronary heart disease-The Framingham Study. Amer J Med 1977; 62: 707-14.

${ }^{21}$ Goldbourt U, Medalie JH. High density lipoprotein cholesterol and incidence of coronary heart disease-the Israeli Ischemic Heart Disease Study. Am J Epidemiol 1979; 109: 296-308.

${ }^{22}$ Hulley SB, Rosenman RH, Bawol RD, Brand RJ. Epidemiology as a guide to clinical decisions. The association between triglyceride and coronary heart disease. $N$ Engl J Med 1980; 302: 1383-9.

${ }^{23}$ Rose G, Baxter PJ, Reid DD, McCartney P. Prevalence and prognosis of electrocardiographic findings in middle-aged men. Br Heart J 1978; 40: 636-43.

${ }^{24}$ Cedres BL, Liu K, Stamler J, et al. Independent contribution of electrocardiographic abnormalities to risk of death from coronary heart disease, cardiovascular diseases and all causes. Circulation 1982; 65: 146-53.

${ }^{25}$ Coronary Drug Research Group. Factors influencing long-term prognosis after recovery from myocardial infarction-three-year findings of the Coronary Drug Project. J Chronic Dis 1974; 27: 267-85.

${ }^{26}$ Pedoe HT. Epidemiology and primary prevention of coronary heart disease. Medicine International 1982; 20: 903-6.

${ }^{27}$ Oliver MF. Coronary atheroma and ischaemic heart disease: aetiology and epidemiology. In: Sleight $P$, Jones JV, eds. Scientific foundations of cardiology. London: Heinemann, 1983; 207-12.

${ }^{28}$ Pedoe HT, Clayton D, Morris JN, Bridgen W, McDonald L. Coronary heart-attacks in East London. Lancet 1975; ii: 833-8.

${ }^{29}$ Kinlen LJ. Incidence and presentation of myocardial infarction in an English community. Br Heart J 1973; 35: 616-22.

${ }^{30}$ Armstrong A, Duncan B, Oliver MF, Julian DG, Donald KW, Fulton M, Lutz W, Morrison SL. Natural history of acute coronary heart attacks. A community study. Br Heart J 1972; 34: 67-80.

${ }^{31}$ Heller RF, Chinn S, Pedoe HT, Rose G. How well can we predict coronary heart disease? Findings in the United Kingdom Heart Disease Prevention Project. Br Med J 1984; 288: 1409-11.

${ }^{32}$ Levy RI, Feinleib M. Risk factors for coronary artery disease and their management. In: Braunwald E, ed. Heart disedse-a textbook of cardiovascular medicine, 2nd edition. Philadelphia: Saunders, 1984; 1205-34. 